\title{
Doenças em bovinos confinados - desafios sanitários em um confinamento de grande porte
}

Anderson Lopes Baptista ${ }^{[a]}$, Pedro de Almeida Fonseca ${ }^{[b]}$, Guilherme Lobato Menezes ${ }^{[c]}$, Layane Queiroz Magalhães ${ }^{[d]}$

\begin{abstract}
[a] Médico veterinário pela UNOESTE, especialista em Produção Animal, mestre em Saúde Animal pela Universidade Federal de Uberlândia (UFU). Atua como responsável pela Saúde Animal da Empresa AC Proteína.

[b] Médico veterinário pela Universidade Federal de Minas Gerais (UFMG), MBA em Gestão Estratégica do Agronegócio pela Fundação Getúlio Vargas e Black Belt em Metodologia Lean Six Sigma. Atua como Gerente de Gestão na AC Proteína, responsável pela execução do Sistema Gerencial e Metodologias de Gestão para resultados.

[c] Médico veterinário pela Universidade Católica de Minas Gerais (PUC Minas). Atua como Analista de Gestão na AC Proteína, responsável pela análise e gerenciamento da rotina dos indicadores nas unidades de Minas Gerais.

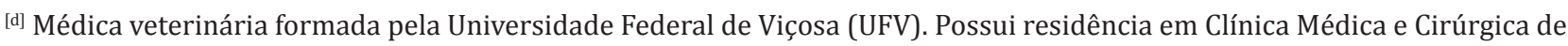
Grandes Animais pela Universidade Federal de Uberlândia (UFU) e atualmente é mestranda pelo Programa de Pós-Graduação em Ciências Veterinárias da UFU, na subárea Clínica Médica e Investigação Etiológica, com ênfase em Doenças Respiratórias dos Bovinos.
\end{abstract}

*Autor correspondente

e-mail: anderson.lopes@acproteina.com.br

\section{Introdução}

O Brasil é reconhecido como o segundo maior exportador mundial de carne bovina (Beef and Veal Meat Exports by Country in 1000 MT CWE - Country Rankings, 2016). No ano 2016 foram abatidos 24.276.178 cabeças, segundo dados do Ministério da Agricultura, Pecuária e Abastecimento (Mapa). Apesar deste destaque no cenário mundial, o país ainda apresenta uma atividade heterogênea nos sistemas de produção e mecanismos de gestão. Coexistem dois subsistemas de produção bastante distintos: o primeiro é de alta qualidade,

caracterizado pela adoção de tecnologia avançada e padrões eficientes de gestão e de comercialização; o segundo, de baixa qualidade, baseia-se na produção extensiva, pequena intensidade tecnológica e padrões precários de gestão e de comercialização do gado (Carvalho e Zen, 2017).

O século XXI traz competitividade, e nesse cenário, as empresas precisam inovar, adaptar-se, implementando procedimentos para produzirem mais e melhor, verificando seus pontos fracos e fortes; também devem avaliar seus desempenhos, 
afinal, "o que não é medido não é gerenciado" (Kaplan e Norton, 1997). Em pecuária, a mensuração dos dados de compra e venda de animais, gastos com nutrição, sanidade, mão de obra, entre outros, são de suma importância para realizar a análise de custos e receitas (Moreira et al., 2009).

O sistema de confinamento entra na cadeia pecuária como estratégia para aliviar a carga das pastagens, encurtar o processo de engorda, aumentar a produtividade ou também como modelo de negócio e prestação de serviço em engorda. Neste modelo, a margem do produtor é menor, a estratégia deve ser bem planejada e o desenvolvimento bem gerenciado. Porém quando falamos em saúde animal, por representar $0,5 \%$ dos custos no sistema de confinamento, muito se fala, pouco se faz e quase nada é mensurado. Isso dificulta a identificação dos principais agente causadores de mortalidade em confinamento e ação corretiva sobre eles, pois não consegue-se gerenciar o que não é mensurado.

A AC Proteína escolheu o indicador de mortalidade para gerenciar o processo de saúde animal, que representa a eficiência desta operação, tendo inclusive uma relação causa-efeito com o lucro do negócio. Apesar de representar pequena fatia na composição do custo do boi confinado, as perdas em saúde animal podem ser diretas, através do aumento da mortalidade e morbidade, ou indiretas, como redução de desempenho.

Neste estudo mostraremos as principais causas de morte em bovinos confinados e o trabalho de redução da mortalidade no período de três anos atuando nos desafios sanitários, bem como seus impactos financeiros e o porquê de termos adotado essa métrica como um indicador a ser gerenciado.

\section{Discussão}

\section{Resultados Operacionais}

As causas de morte em confinamento variam em função do desafio encontrado na região onde o mesmo é instalado. Em análise feita de 2014 a 2016, foram apontadas como principais causas a pneumonia, clostridioses e causas acidentais (Figura 1), porém essas não são imutáveis, graças ao trabalho de melhoria contínua realizado durante os anos.

0 trabalho de melhoria contínua permitiu uma redução de $50 \%$ na mortalidade do confinamento em 2016 comparado ao ano de 2014, conforme a Figura 2. Isso foi possível através da adoção de medidas estratégicas que agiram na causa raiz do problema.

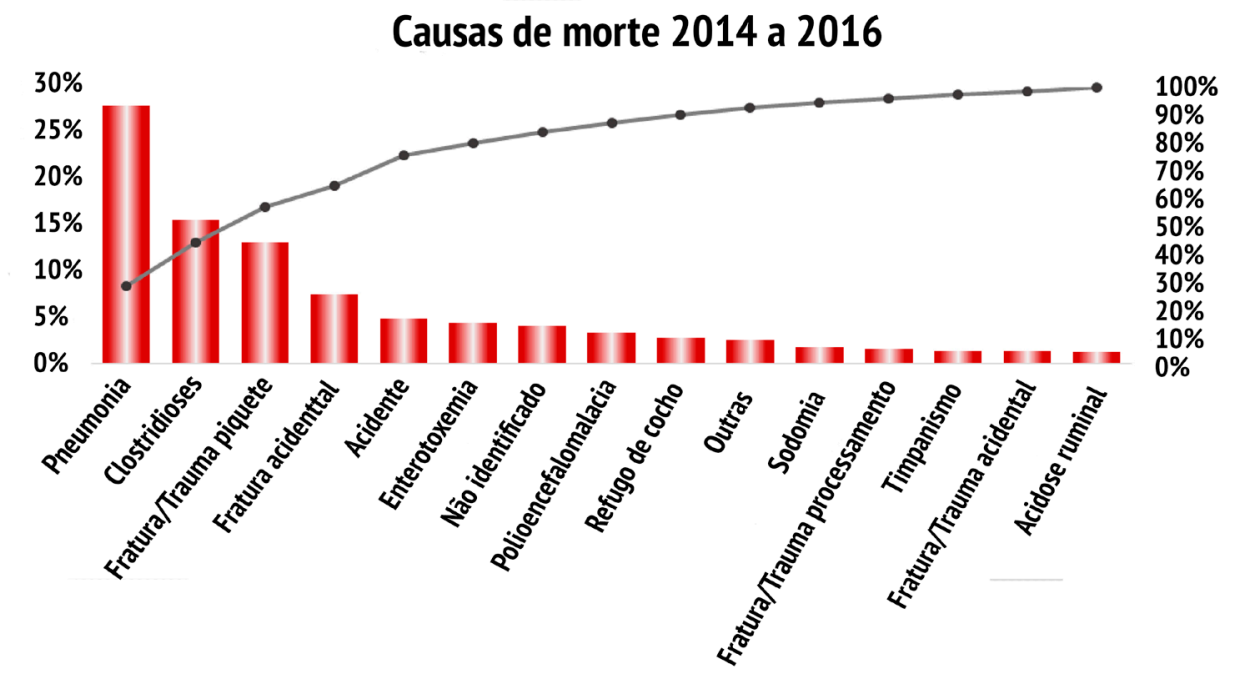

Fonte: Sistema de Gestão/AC Proteína.

Figura 1 - Causas de morte 2014 a 2016. 


\section{Comparação 2014 a 2016}

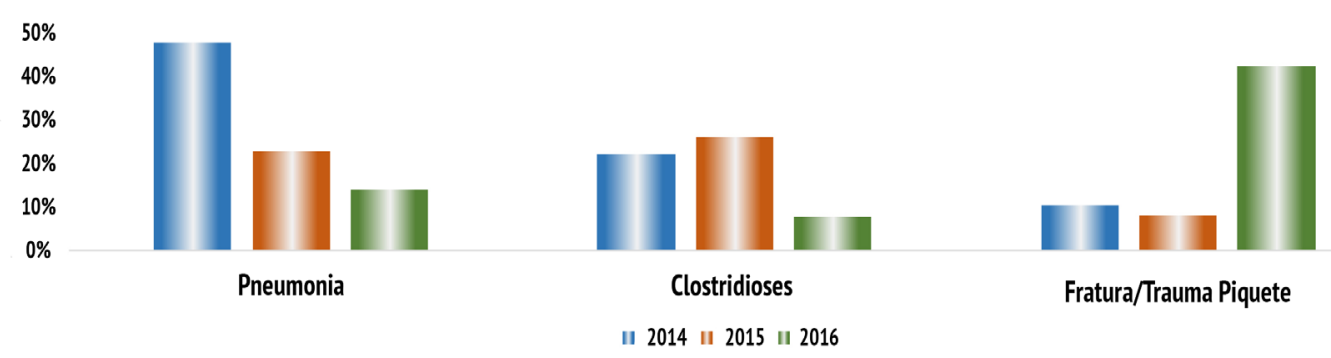

Fonte: Sistema de Gestão/AC Proteína.

Figura 2 - Comportamento da causas de morte - 2014, 2015 e 2016.

Em 2014, a meta de mortalidade era 0,45\% e o ocorrido $0,78 \%$, apresentando um desvio de $0,36 \%$. Através da Análise de Pareto, percebeu-se que as causas de morte concentraram-se em três grupos: DRB, Clostridiose e Acidental. Neste contexto, a DRB representou $37 \%$ das causas de morte.

Foi feita análise de processo e identificados alguns fatores de risco, dentre eles: distância percorrida e tempo de viagem, tendo os animais que viajaram mais de $500 \mathrm{~km}$ ou com tempo de viagem superior a 8h maior predisposição a adoecerem; época do ano (durante o período de secas aumenta o nível de poeira e a amplitude térmica é maior); escore de condição corporal 1 e 2 (escala de 1 a 5); e origem, pois animais oriundos de compra apresentaram mortalidade de $0,66 \%$ frente aos $0,28 \%$ de animais de recria própria.

Observou-se, também, que as medicações estavam concentradas na segunda semana de confinamento e que o pico de mortalidade ocorria na terceira semana (Figura 3). Coutinho (2004) relata maior incidência de doença respiratória nas três primeiras semanas de confinamento. Do $1^{\circ}$ ao $7^{\circ}$ dia ocorrem $10 \%$ das manifestações clínicas. Até o $15{ }^{\circ}$ dia são observadas $60 \%$ das manifestações. Até o $21^{\circ}$ dia, $80 \%$ das manifestações. A partir do $30^{\circ}$ dia até o abate, a manifestação é de $10 \%$.

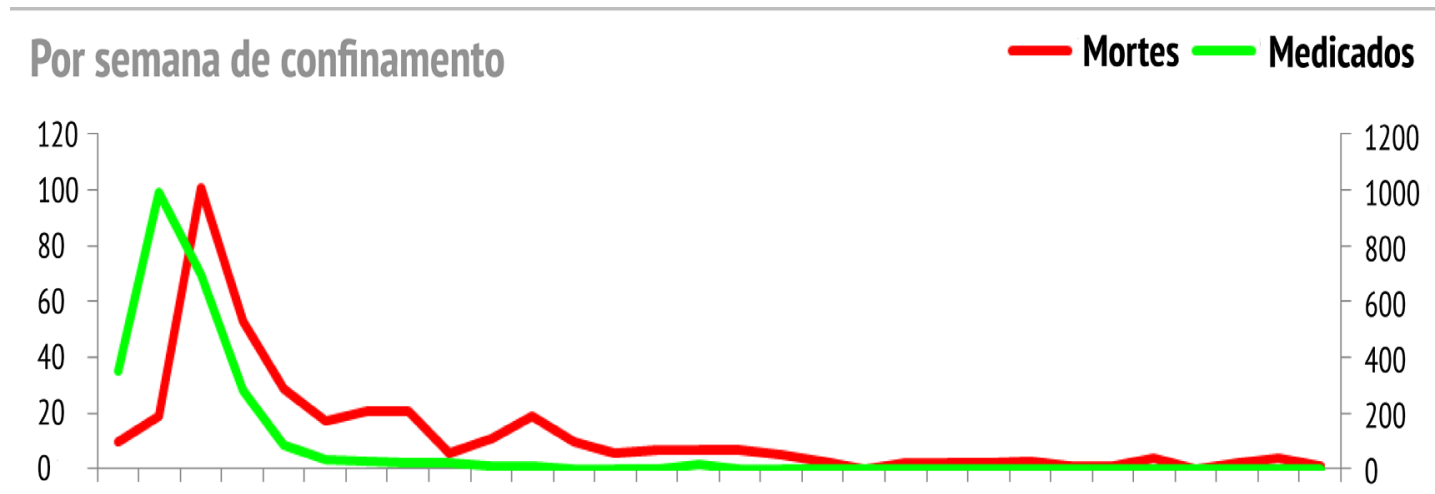

123456789101112131415161718192021222324252627282930

Fonte: Sistema de Gestão/AC Proteína.

Figura 3 - Distribuição de mortes e medicações por semana de confinamento.. 
Neste período foi identificado um menor número de medicações nos dias de sexta-feira, sábado e domingo devido à redução do quadro de funcionários. Consequentemente, ocorriam picos de mortes no início da semana, reduzindo conforme o nível de medicações normalizasse.

Foram adotadas algumas ações, entre elas a construção de uma estrutura de recepção e descanso dos animas, irrigação dos piquetes, adoção de protocolo sanitário baseado em fatores de risco, contratação de dois colaboradores para compor a equipe de ronda sanitária e estabilização das medicações por dia da semana.

Já em 2015, houve uma redução significativa na mortalidade, caindo de $0,78 \%$ para $0,46 \%$, devido à padronização de processos no controle de DRB. No ano de 2015, após a redução das mortes por DRB, ficaram evidentes as mortes por clostridiose, que representaram 28\% de todas mortes do confinamento.

Os diagnósticos foram baseados em exames macroscópicos denecropsia, ondeforamidentificados enterite hemorrágica, ruminite e abomasite, além de quadros de queda de consumo. Foi coletado material para análise laboratorial e identificado Clostridium perfringens. Alterações na microbiota ruminal, em decorrência de mudanças bruscas na alimentação, fornecimento de dietas ricas em carboidratos e pobre em fibras, dentre outros fatores, levam à multiplicação do agente em proporções logarítmicas causando a doença (Lobato et al., 2013).

Para monitorar o tamanho da fibra na dieta foi adotada rotina de avaliação da estratificação de partículas, segundo adaptação da metodologia das peneiras sequenciais do Penn State Particle Size Separator, proposta por Lammers et al. (1996), definindo-se a porcentagem de material de diâmetro superior a $38 \mathrm{~mm}$ (peneira 1), intermediário de 38 a $19 \mathrm{~mm}$ (peneira 2) e de 19 a 7,9 $\mathrm{mm}$ (peneira 3), e inferior a 7,9 mm.

Além disso, foi adotado aumento da proporção de volumoso na dieta e rotina de avaliação do escore de fezes para identificação da ocorrência de acidose. Segundo Ferreira et al. (2013), existe correlação do escore fecal de bovinos com distúrbios alimentares, como acidose, e a caracterização fecal pode dar informações rápidas para a tomada de decisão no manejo alimentar nutricional.
No ano 2016, a meta de mortalidade foi alcançada com o resultado de 0,31\%, que representa a efetividade das ações adotadas nos anos anteriores, principalmente contra DRB e clostridiose. Neste ano, as mortes por causas acidentais foram identificadas na Análise de Pareto como o principal fator de influência no indicador e, por isso, as ações foram direcionadas para a resolução deste problema. Foi implantado o manejo racional dos animais através do Creating Connections, programa desenvolvido nos Estados Unidos pelo médico veterinário Tom Neffsinger, que mostra uma nova forma de manejar o gado, criando uma base de confiança entre o homem e o animal apenas com a linguagem corporal e sem o uso de objetos. Além deste programa, foi construído um hospital veterinário com objetivo de melhorar o manejo dos animais doentes e a acurácia no diagnóstico, e evitar acidentes nos piquetes de confinamento.

\section{Análise Econômico-Financeira}

A análise de viabilidade econômico-financeira deste projeto de melhoria contínua foi feita na avaliação de dois cenários de resultados: anterior ao projeto e atual. Cada processo foi estratificado, considerando seus drivers, variáveis produtivas e o custo ou ganho financeiro.

No âmbito de custos de operação, a utilização da metafilaxia aumentou o custo animal, ponderado em $\mathrm{R}$ \$ 4,07 em relação ao ano de 2014, quando não se usava esse protocolo. 0 custo animal com mão de obra também aumentou em $\mathrm{R} \$ 0,57$ por animal, devido à contratação de dois colaboradores. Observou-se uma redução de custo por morbidade, que passou de $11,6 \%$ para $9,2 \%$, representando um saldo positivo de $\mathrm{R} \$ 2,56$ por animal.

Contribuindo no aumento de receita operacional, observou-se diferença de 0,026 g de ganho diário de carcaça dos animais sadios em relação aos doentes, representando $\mathrm{R} \$ 29,56$ por animal que deixou de ficar doente. Isso representou $\mathrm{R} \$ 0,72$ por animal abatido por ano.

Nos processos que refletiram redução de desperdícios, a implantação de boas práticas de manejo resultou em uma redução de traumas de carcaça de $4,11 \%$ para $3,11 \%$, representando um saldo positivo de $\mathrm{R} \$ 0,10$ a mais por animal abatido. 
Ainda nos processos de redução de desperdícios, houve uma redução de $0,47 \%$ na mortalidade, equivalente a um saldo positivo de $\mathrm{R} \$ 12,48$ a mais por animal, confirmando ser o principal driver em saúde animal com impacto financeiro representativo.

Com a construção do hospital veterinário, esperase uma redução de mortes por acidente, e por isso foi estipulada redução desse índice de $0,14 \%$ para 0,08\%, compondo a meta total do ano de 2017. Esta melhoria representa uma redução de desperdícios de $\mathrm{R} \$ 1,70$ por animal.

No período total do projeto, foram feitos investimentos na construção de uma estrutura de recepção de animais, no valor de $\mathrm{R} \$ 228.889$, na instalação do sistema de irrigação de piquetes, no valor de $\mathrm{R} \$ 272.340$, e na construção do hospital veterinário, no valor de $\mathrm{R} \$ 180.000$, totalizando $\mathrm{R} \$$ 681.229 investidos em instalação.

A Tabela 1 representa o demonstrativo de saldo por animal de cada processo e a projeção para 100.000 animais, que é a capacidade de abate anual deste confinamento. Com todas as melhorias realizadas, essas ações deixaram um saldo positivo de $\mathrm{R} \$$ 1.291.045/ano. Considerando este valor, obtevese um payback de 6,3 meses dos investimentos realizados.

Tabela 1 - Demonstrativo de saldo por processos considerados na viabilidade da saúde animal

\begin{tabular}{lcc}
\hline Processos & Saldo por animal (R\$) & ${ }^{*}$ Saldo anual (R\$) \\
\hline Aumento de receita GDC & 0,72 & 71.714 \\
$\begin{array}{l}\text { Redução de desperdício } \\
\text { por trauma de carcaça }\end{array}$ & 0,10 & 9.567 \\
$\begin{array}{l}\text { Redução de desperdício } \\
\text { por mortalidade }\end{array}$ & 12,48 & 1.247 .733 \\
$\begin{array}{l}\text { Redução de desperdício } \\
\text { por morte por acidente }\end{array}$ & 1,70 & 170.300 \\
$\begin{array}{l}\text { Redução de custo por } \\
\text { medicações }\end{array}$ & 2,56 & 255.975 \\
$\begin{array}{l}\text { Aumento de custo por } \\
\text { metafilaxia }\end{array}$ & $-4,07$ & -407.043 \\
Aumento de custo MD0 & $-0,57$ & -57.200 \\
\hline Total & 12,91 & 1.291 .045
\end{tabular}

Nota: * Projetado para 100.000 animais.

Fonte: Sistema de Gestão/AC Proteína.

\section{Considerações finais}

Devemos identificar lacunas em todos os processos produtivos através da utilização de metodologia de gestão, associada a conhecimentos técnicos e liderança, e buscar constantemente a melhoria de resultados. Em saúde animal, observamos uma grande oportunidade de ganhos financeiros e operacionais, confirmando a importância deste processo na cadeia de produção de carne bovina.

\section{Referências}

Beef and Veal Meat Exports by Country in 1000 MT CWE Country Rankings. 2016 [acesso 13 fev 2016]. Disponível em: https://tinyurl.com/qey4knc.

Carvalho TB, Zen S. A cadeia de Pecuária de Corte no Brasil: evolução e tendências. Rev iPecege. 2017;3(1):85-99.

Coutinho AS. Mannheimiose pneumônica experimentalmente induzida em bezerros pela Mannheimia (Pasteurella) haemolytica A1- cepa D153: achados do exame físico, hemograma e swabs nasal e nasofaringeano [tese de doutorado]. Botucatu: Universidade Estadual Paulista; 2004.

Ferreira SF, Guimarães T, Moreira K, Alves V, Lemos B, Souza F. Caracterização fecal de bovinos. Rev Cient Elet Med Vet. 2013;20(1):1-22.

Kaplan R, Norton D. A estratégia em ação: balanced scorecard. Rio de Janeiro: Campus; 1997.

Lammers BP, Buckmaster DR, Heinrichs AJ. A simple method for the analysis of particle sizes of forage and total mixed rations. J Dairy Sci. 1996;79(5):922-8.

Lobato FCF, Salvarani FM, Gonçalves LA, Pires PS, Silva ROS, Alves GG, et al. Clostridioses dos animais de produção. Vet e Zootec. 2013;20(Ed Comemorativa):29-48.

Moreira SA, Thomé KM, Ferreira PS, Botelho Filho FB. Análise econômica da terminação de gado de corte em confinamento dentro da dinâmica de uma propriedade agrícola. Custos e @gronegócio on line 2009;5(3):132-52. 